\title{
Molecular tools for monitoring harmful algal blooms
}

\author{
Linda Medlin
}

Received: 4 September 2012 / Accepted: 11 September 2012 /Published online: 3 July 2013

(C) Springer-Verlag Berlin Heidelberg 2013

The world's oceans cover $70 \%$ of the Earth's surface, and their dominant populations, both numerically and biomasswise, belong to microscopic protists and prokaryotes. The marine phytoplanktons are major components of both groups and are, by definition, high dispersal taxa with large population sizes. Small photosynthetic organisms are responsible for the bulk of primary production in oceanic and neritic waters. Microalgae in marine and brackish waters of Europe regularly cause harmful effects, considered from the human perspective, in that they cause economic damage to fisheries and tourism. Cyanobacteria cause similar problems in freshwaters. These episodes encompass a broad range of phenomena collectively referred to as 'harmful algal blooms' (HABs). For adequate management of these phenomena, monitoring of microalgae is required.

However, present day monitoring is time consuming and based on morphology as determined by light microscopy, which may be insufficient to give definitive species and toxin attribution. The public perception of harmful algal blooms is often that of eutrophication, where increased nutrient loading results in large biomass blooms, which have a consequent effect on water quality (anoxia) and the ecosystem through substantial alterations in both the benthic and pelagic ecology. All too often, events that directly or indirectly relate to increased nutrients, such as dead zones in the northern Gulf of Mexico, a huge clean-up operation following an Enteromorpha bloom off Qingdao in time for the Olympic

Responsible editor: Philippe Garrigues

L. Medlin $(\square)$

Marine Biological Association of the UK, The Laboratory,

Citadel Hill,

Plymouth PL1 2PB, UK

e-mail: 1km@mba.ac.uk sailing competition in 2008 or the knock-on effects of hydrogen sulphide toxicity caused by rotting vegetation following extensive growth of both macro- (Ulva, Brittany coast of France) and micro- (Phaeocystis, North Wales, UK) algae, find substantial room in the popular press.

What is less well-known is that HABs caused by toxinproducing algae have not only a far more serious impact on the quality of life, but also they are entirely natural. Anthropogenic impacts on toxic HABs are limited to either their global spread through potential transport vectors, such as ballast water of ships, or because of alterations in the coastline resulting from the construction of harbours and marinas, particularly around the Mediterranean coast, which promotes the development of chronic dinoflagellate blooms, such as the paralytic shellfish poisoning (PSP) toxin producer Alexandrium.

The global scale of toxin-producing microalgae should not be underestimated. For example, the most serious would be the numbers of human intoxications with ciguatera, caused by the dinoflagellate Gambierdiscus, is currently estimated at some 50,000 per year. Every year, one to two human deaths are linked to the ingestion of PSP toxins. Although these problems are restricted to the tropical/warm temperate sphere of the globe, it demonstrates the urgent need to be able to monitor and prevent toxic HAB events. In Europe, this is effected through a series of directives that require coastal member states to monitor water for toxinproducing species and their toxins in shellfish. Starting with the EU Shellfish Hygiene Directive 91/492/EEC, a series of directives was issued to include newly discovered toxins and stipulating the methods of analysis and maximum permitted levels in shellfish. The most important of these would be 2002/225/EC and 2074/2005 (pertaining to toxin levels and analysis and methods) and more recently 15/2011 
(analysis methods). The natural occurrence of toxinproducing algae, and the continual human demand for shellfish consumption, means that their monitoring is here to stay.

The cost of this monitoring of plankton and toxins is enormous. Although there is limited 'hard' information on the economic impact of HABs, a relatively recent study in the USA (Anderson et al. 2000) has estimated, on a national basis, that:

- The cost of monitoring is equivalent to $5 \%$ annual shellfish industry turnover.

- The cost of lost harvest and damaged product caused by contamination with biotoxins is $5 \%$ of industry turnover.

- The public health costs caused by lost working days, hospitalisations, etc. add another $5 \%$ of annual turnover.

In Europe, similar information is also difficult to uncover, but the context is well set in that if one takes the case of Ireland, the shellfish aquaculture production currently runs at $€ 47$ million annually (Bowne et al. 2007) and the budget for the Irish National Biotoxin and Toxic Phytoplankton monitoring programme, carried out under the auspices of the Food Safety Authority of Ireland and operated through the Irish Marine Institute, is $€ 1.7$ million, representing $3.5 \%$ of annual industry turnover. Similarly, Scottish shellfish production is valued at $\sim £ 20$ million, the most part of which is through culture of the edible mussel Mytilus edulis, and the monitoring programmes, run by the Food Standards Agency Scotland, has a budget of just under $£ 2$ million. Figures for the cost of harvest closures and destroyed stock are unavailable, but the parity with the monitoring cost with that of the USA allows a confidence in the data provided by Anderson et al. (2000).

Clearly, the development of an industry that is both natural and sustainable, but which has such a heavy financial burden, requires all the assistance possible in order to overcome such a 'natural hazard' as toxic HABs, as the (natural) problems caused by toxicity will never go away. Approximately, 2,000 water samples are analysed annually in Ireland as part of the National Monitoring Programme. This requires a staff of four people, augmented slightly during the busy summer months. Most samples are scanned for toxic/harmful species but samples from ten sites (out of a total of $\sim 60$ ) are analysed for their total phytoplankton community. Light microscopy is the routine analysis method, each sample requiring ca. $2 \mathrm{~h}$ on average to examine. Occasionally, some samples for Pseudo-nitzschia are analysed using fluorescence in situ hybridization (FISH). Comparable figures for other monitoring programmes are annual throughputs of 1,000 samples (Scotland), 5,000 samples (REPHY, France) and 6,000 samples (Galicia, Spain). These figures reflect a work rate of processing some 20 samples per week per person. The number of man-hours involved in the monitoring process is clearly enormous. Molecular techniques, which are faster and more reliable, would reduce the number of inevitable mistakes caused by human error that is an ever present facet of this type of work.

Efficient use of molecular techniques for monitoring phytoplankton will significantly increase sample throughput rate over conventional light microscopy methods. Couple this with the benefits of increased accuracy and the advantages of the molecular approach far outweigh any required capital investment. Of particular relevance are the situations with respect to Pseudo-nitzschia, which cannot be identified to species level using light microscopy, and Alexandrium, another genus with which it is also virtually impossible to identify accurately to species using this technique. With respect to the latter, a case in point would be the situation in Cork Harbour, where for over a decade, it was recognised that Alexandrium tamarense was the PSP-producing species (FAO, 2004). Introduction of the use of molecular techniques quickly resolved that Alexandrium minutum was in fact the toxin producer, and that the co-occurring $A$. tamarense was the non-toxic Gp III (Western European) form (Touzet et al. 2008). This is a level of accuracy essential if predictive models are to be produced that can forecast toxic blooms and that can then (and only then) allow their mitigation. Invariably, these models are produced using data derived from monitoring programmes.

As regards to toxin-testing capability, during the course of the project, the European Commission published a new directive $(15 / 2011)$ that changed the official reference method for the analysis of biotoxins in shellfish from the mouse and rat bioassay to a chemical method. The validated technique of liquid chromatography (LC) mass spectrometry (MS) should now be applied as the reference method for the detection of lipophilic toxins and used as matter of routine, both for the purposes of official controls at any stage of the food chain and own-checks by food business operators. Of particular relevance is the statement that LC MS/MS is the reference method for okadaic acid and derivatives, yessotoxins, pectenotoxins and azaspiracids. Antibodies are now available for most of the major groups of algal toxins, and the adaptation of toxin/antibody recognition techniques to fast throughput methodology is an attractive alternative to LCMS.

The advent of molecular biological techniques has greatly enhanced our ability to analyse all populations, not just the marine phytoplankton. Their small size and paucity of morphological markers, the inability to bring many into culture and the difficulty of obtaining samples for long-term seasonal studies in open ocean environments have hampered our knowledge of phytoplankton diversity and population structure (Medlin and Kooistra 2010). These techniques are slowing making inroads into monitoring for toxic algae in 
terms of monitoring for the presence of a species and the toxins they produce.

One approach that is extensively in such studies is to identify species by specific molecular probes. Probes are short oligonucleotides of normally 16-24 bp length that are $100 \%$ homologous only to a complementary sequence in a gene of the species of interest and differ by at least one position to all other organisms. In hybridisation experiments, these probes can therefore be used to identify species of interest by binding to the target's sequence and later detection by a probe-attached label, e.g., digoxigenin or a fluorochrome, such as fluorescein. In qPCR experiments, these probes used as PCR primers can be used to estimate cell numbers. The application range of these probes and primers extends from answering ecological questions like species composition and its change through space and time to the development of an early warning system for harmful algal blooms using probes for toxic species.

In total, 18 papers were accepted and included in this special issue, showing a variety of molecular and cytometric techniques for monitoring of toxic algae ranging from FISH probes to qPCR to antibody ELIZA tests to "image in flow" cytometry and from laboratory validation to field validation. 11 of these papers have originated from the EU FP7 project microarrays for the detection of toxic algae (MIDTAL). In that project, rapid species identification using rRNA genes was developed for use in a species microarray or phylochip. rRNA regions can be used for probe design to recognise species or even strains. MIDTAL also developed a toxinbased microarray that includes antibody reactions to specific toxins produced by these microalgae because even when cell numbers are low and below the detection limit of the phylochip, toxins can be present and can accumulate in the shellfish. Microarrays are the state of the art technology in molecular biology for the processing of bulk samples for detection of target RNA/DNA sequence. Existing rRNA probes and antibodies for toxic algal species/strains and their toxins were adapted and optimised for microarray use. The purpose of MIDTAL is to support the common fisheries policy and aid the national monitoring agencies by providing new rapid tools for the identification of toxic algae and their toxins so they can comply with ECC directives (EU) no., 15/2011 and converted microarray signals to cell numbers to reduce the need for the mouse bioassay. The project MIDTAL does not, at present, seek to alter this legislation: the toxin analysis capability of the microarray can still be used satisfactorily as a method for end-product testing. The project MIDTAL has been an endeavour to improve national monitoring capabilities for toxin-producing plankton species, and their toxins, in water samples.
Overall, this special issue exemplifies the need for approaches other than just light microscopy to identify toxic algae and has shown alternative methods than the mouse bioassay and HPLC which are essential to produce reliable assessments and predictions on the occurrence of toxic algal blooms, and these methods are not currently accepted as valid methods for monitoring toxic events by the EU but which can be and implemented by water resource managers to ensure that validation should be carried out.

\section{References}

Anderson DM, Kaoru Y, A.W. White AM (2000) Estimated annual economic impacts from harmful algal blooms (HABs) in the United States. Woods Hole Oceanographic Institution Technical Report WHOI-2000-11, 97 pp.

Browne R, Deegan B, O'Carroll T, Norman M, O'Cinneide M (2007) Status of Irish aquaculture 2006. Marine Institute, Dublin

FAO (2004) Marine biotoxins. FAO Food and Nutrition Paper 80, Food and Agriculture Organization of the United Nations, Rome.

Medlin LK, Kooistra, WHCF (2010) Methods to estimate the diversity in the marine photosynthetic protist community with illustrations from case studies: a review. Special issue of diversity "Biological Diversity Assessed by Molecular Methods," 2: 973-1014.

Touzet N, Franco JM, Raine R (2008) PSP toxin analysis and discrimination of the naturally co-occurring Alexandrium tamarense and A. minutum in Cork Harbour, Ireland. Aq Micro Ecol 51:285-299

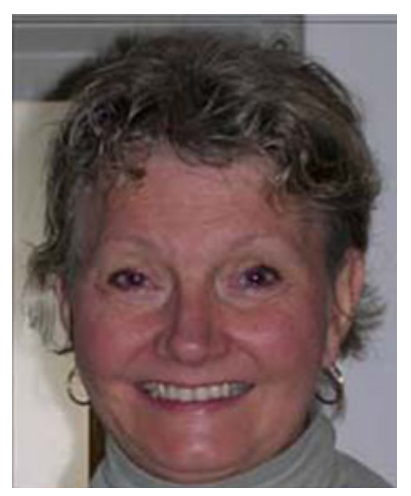

Linda K. Medlin was most recently a research scientist at the University of Pierre and Marie Curie Paris at the Observatoire Océanologique de Banyuls-sur-Mer, France. She holds a Research Fellow Appointment at the Marine Biological Association of the UK where she co-ordinated the FP7 EU project MIDTAL. She obtained her Ph.D. from Texas A\&M University in 1983. Professor Medlin's research interests are in the areas of the evolution of the marine phytoplankton, and she has produced phylogenies and molecular clocks for the diatoms, dinoflagellates and haptophytes. She has also produced a revised taxonomy for the diatoms and the haptophytes from these molecular phylogenies. Her recent research efforts have been directed towards the making of species specific probes for making early warning systems for toxic algae and applying them in a microarray and biosensor format and for making phylochips for the analysis of marine biodiversities using microarrays. She has published over 220 research papers including 35 chapters in books. She has participated in over $15 \mathrm{EU}$ grants and many other national funding proposals from the UK and Germany 